\title{
Neumomediastino espontáneo
} Spontaneus pneumomediastinum

\author{
F. J. Ruiz-Ruiz, A. Sampériz, T. Rubio, F. Escolar
}

\section{RESUMEN}

El neumomediastino espontáneo (definido por la presencia de aire libre en el mediastino) es una entidad infrecuente que se presenta en ausencia de enfermedades o factores precipitantes. Generalmente afecta a varones jóvenes y sanos. El dolor torácico, la disnea o ambos son los síntomas más frecuentes. Otros síntomas y signos son el enfisema subcutáneo y el signo de Hamman. La radiografía simple de tórax establece el diagnóstico en un alto porcentaje de pacientes. El tratamiento conservador está indicado en estos pacientes y tiene un pronóstico favorable. Las recidivas son infrecuentes.

Palabras clave. Neumomediastino espontáneo. Dolor torácico. Diagnóstico. Tratamiento.

\begin{abstract}
Spontaneous pneumomediastinum (defined by the presence of free air in the mediastinum) is an infrequent entity that is presented in the absence of diseases or precipitating factors. It generally affects young and healthy males. Thoracic pain, dyspnea, or both, are the most frequent symptoms. Other signs and symptoms are subcutaneous emphysema and Hamman's sign. Simple radiography of the thorax allows for the diagnosis in a high percentage of patients. Conservative treatment is indicated in these patients and has a favourable prognosis. Relapses are infrequent.
\end{abstract}

Key words. Spontaneous pneumomediastinum. Thoracic pain. Diagnosis. Treatment.
1. Servicio de Medicina Interna. Hospital Reina Sofía. Tudela. Navarra.

Aceptado para su publicación el 6 de febrero de 2006.

\section{Correspondencia:}

Francisco J. Ruiz Ruiz

Servicio de Medicina Interna

Hospital Reina Sofía

Ctra. de Tarazona Km 4

31500 Tudela (Navarra)

Tfno. 848434000

Fax. 848434111

E-mail: fruiz@comz.org 


\section{INTRODUCCIÓN}

El neumomediastino se define como la presencia de aire en el mediastino. Las causas más frecuentes implicadas en su aparición son heridas o traumatismos penetrantes, infección por bacterias productoras de gas o rotura esofágica secundaria a vómitos o exploraciones endoscópicas. El pneumomediastino espontáneo es el que se produce sin factores desencadenantes aparentes, afectando principalmente a varones jóvenes. Este último constituye una patología poco frecuente, con una incidencia variable que se cifra, como media, en 1 por cada 10.000 ingresos hospitalarios y supone en torno al 1\% del total de casos de pneumomediastino. Se ha sugerido que esta baja incidencia fuera consecuencia del infradiagnóstico de esta patología y del tipo de población asignada a cada hospital, pues las cifras descritas en diferentes series oscilan entre 1 por cada 800 a 1 por cada 42.000 ingresos ${ }^{1,2}$.

Debido a su escasa frecuencia y a que sus síntomas pueden conducir a diagnósticos erróneos y exploraciones costosas, creemos interesante recordar esta entidad. Para ello presentamos el siguiente caso clínico y a continuación realizamos una breve revisión de este proceso de diagnóstico sencillo en la mayoría de casos asociado a buen pronóstico.

\section{CASO CLÍNICO}

Se trata de un paciente de 16 años, sin antecedentes patológicos de interés, que encontrándose previamente bien comienza de madrugada con dolor en región precordial de tipo punzante, que le despierta, y que aumenta con los movimientos respiratorios y al inclinarse hacia adelante. Refiere odinofagia. En días previos había presentado dolor abdominal y astenia, motivo por el que consultó a su médico de cabecera, habiendo sido diagnosticado de un proceso viral inespecífico.

A la exploración física presenta un hábito asténico, presión arterial de 110/60 $\mathrm{mmHg}$, temperatura axilar de $36^{\circ} \mathrm{C}$ y un buen estado general y de hidratación, así como una buena coloración de piel y mucosas. La faringe aparecía enrojecida. A la auscultación cardiaca presentaba tonos rítmi- cos a 82 latidos por minuto y crepitantes secos en los diferentes focos cardiacos. La auscultación pulmonar no mostró alteraciones patológicas, como tampoco mostraron signos patológicos la exploración abdominal y el resto de la exploración física. Se realizó un ECG que no mostró alteraciones en el ritmo ni en la repolarización. Los resultados del hemograma y bioquímica (incluyendo CK y troponina) estaban en rango de normalidad. La radiografía realizada mostraba la existencia de un neumomediastino (Fig. 1). Se inició tratamiento con oxigenoterapia, reposo y analgésicos, mejorando progresivamente la clínica que presentaba el paciente al ingreso. Al tercer día el paciente se encontraba asintomático, por lo que fue dado de alta. Quince días después el control radiológico efectuado mostró la resolución completa del neumomediastino.

\section{DISCUSIÓN}

Como se ha mencionado anteriormente el neumomediastino espontáneo es un proceso poco frecuente que afecta, principalmente, a varones jóvenes sin factores desencadenantes aparentes. La relación hombre/mujer se estima en $8 / 1$, cifra similar a la observada en casos de neumotórax espontáneo ${ }^{1}$. Conocer la incidencia real de este proceso es difícil debido a que la mayor parte de la literatura médica recoge la descripción de casos aislados, y las series de casos recogidas apenas hacen referencia a unas pocas decenas de pacientes. Estudios recientes realizados en nuestro entorno, y referenciados a un área poblacional de 250.000 habitantes, muestran una incidencia de 4,5 casos/año, constituyendo el $0,005 \%$ de las urgencias hospitalarias atendidas en un año ${ }^{3}$.

Diferentes factores se han implicado en su aparición, tales como asma, cetoacidosis diabética, quimioterapia, radioterapia, drogas inhaladas, aumentos bruscos en la presión intraalveolar y actividades relacionadas con la maniobra de Valsalva ${ }^{46}$. Otros autores han encontrado relación con la práctica de terminados deportes entre los que destacaría, por su mayor asociación con el cuadro, el submarinismo ${ }^{7}$. Recientemente se han comunicado casos en relación al consumo de drogas de diseño como 
el éxtasis o "speed"2. También se han comunicado casos en adolescentes con trastornos de la conducta alimentaria, principalmente anorexia nerviosa ${ }^{8}$.
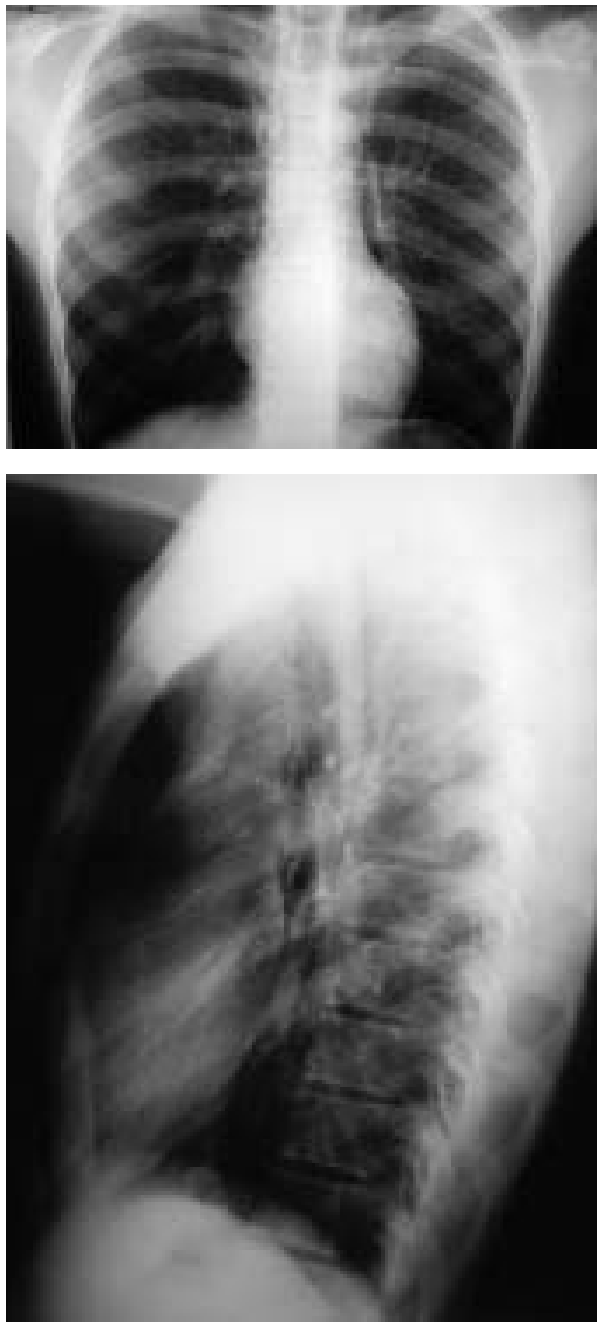

Figura 1. La radiografía de tórax resulta diagnóstica en la mayoría de casos al observarse la presencia de aire delimitando el corazón y resto de estructuras mediastínicas.

Si bien el cuadro clínico había sido descrito con anterioridad por Laennec (a comienzos del siglo XIX) y posteriormente por Hamman (en 1939), fue Macklin quien describió su fisiopatología en 1944. Se explicaría por la rotura de alvéolos terminales y la disección que el aire liberado produciría en la vasculatura pulmonar, accediendo al mediastino a través del hilio ${ }^{1,6}$.

Clínicamente se caracteriza por la aparición de dolor torácico agudo junto con disnea, disfagia, odinofagia y enfisema subcutáneo. En las diferentes series de casos clínicos publicadas, el dolor torácico es el síntoma con el que, con mayor frecuencia, se manifiesta el neumomediastino espontáneo. La presentación como dolor torácico, disnea o la combinación de ambos está presente hasta en el $70 \%$ de los casos. Un signo característico a la exploración es la auscultación de crepitantes secos en los diferentes focos cardiacos que nuestro paciente presentaba, y que se denomina signo de Hamman, y que traduce en la existencia de aire en el saco pericárdico. Si bien su presencia es altamente sugestiva del cuadro, sólo está presente en menos de la mitad de los $\operatorname{casos}^{4,9,10}$. En un porcentaje similar puede observarse la presencia de enfisema subcutáneo, si bien en algunas series este porcentaje asciende hasta el $80 \%{ }^{11}$. En la serie de Campillo-Soto ${ }^{3}$, una de las mayores publicadas hasta la fecha, con 36 casos, la presencia de disnea, dolor torácico o ambos se presentó en el $70 \%$ de los casos, apareciendo otros síntomas, tales con dolor cervical, tos y disfonía en un 14, 11 y $5 \%$ respectivamente. Los signos que se presentaron con mayor frecuencia en esa serie fueron la crepitación cervical y los sibilantes respiratorios, ambos descritos en un 14\% de los casos. Sólo 2 pacientes presentaron los signos de Hamman ${ }^{3}$.

Esta variabilidad clínica en su forma de presentación y lo infrecuente de la misma requiere un alto índice de sospecha para su diagnóstico. Éste viene dado por la radiografía de tórax, donde puede apreciarse la existencia de aire alrededor del corazón, de los grandes vasos y del mediastino, delimitado por la pleura. En proyección lateral, que suele ser más adecuada para el diagnóstico, pueden observarse cantidades variables de aire en mediastino anterior, alrededor de aorta ascendente e incluso alrededor del timo (signo de la aleta tímica). Otros signos que se han relacionado con la presencia de 
neumomediastino son el signo de la $\mathrm{V}$ de Naclerio, que consiste en la presencia de aire en la zona costovertebral adyacente al hemidiafragma y la columna vertebral, y la coexistencia en la radiografía de enfisema subcutáneo ${ }^{12}$.

En la mayoría de casos, la radiografía de tórax es suficiente para establecer el diagnóstico, si bien se han descrito casos con radiografía aparentemente normal donde el diagnóstico lo ha establecido la tomografía computarizada (TAC), correspondiendo estos casos a aquellos con una menor cuantía de aire en mediastino ${ }^{13}$.

El estudio del cuadro clínico puede completarse con otras técnicas (TAC, esofagograma, electrocardiograma, ecocardiograma...) que permitan establecer un diagnóstico diferencial o establecer otras posibles causas. El diagnóstico diferencial incluye todas aquellas etiologías cardiacas, pulmonares, esofágicas y músculoesqueléticas capaces de provocar dolor torácico, disnea o ambos.

El tratamiento se basa en reposo, oxigenoterapia y analgesia. La estancia media hospitalaria, de los casos que ingresan, oscila entre los 2-7 días, según las series publicadas. En la gran mayoría de casos la evolución es satisfactoria, siendo infrecuente su recurrencia ${ }^{11}$.

En el caso presentado, el paciente mostró una semiología y evolución típicas, objetivándose el signo de Hamman característico del neumomediastino espontáneo, aunque no siempre presente. A pesar de ello deben tenerse en cuenta formas de presentación diferentes a la descrita y mencionadas en esta revisión.

Como conclusión puede afirmarse que el neumediastino espontáneo debería figurar entre los diagnósticos diferenciales a plantear en un varón joven que consulta por dolor torácico. Generalmente la radiografía de tórax es suficiente para establecer el diagnóstico. En la gran mayoría de casos la evolución es buena con tratamiento sintomático, siendo excepcional la recidiva.

\section{BIBLIOGRAFÍA}

1. Gerazounis M, Athanassiadi K, Kalantzi N, MousTARDAS M. Spontaneous pneumomedi- astinum: a rare bening entity. J Thorac Cardiovasc Surg 2003; 126: 774-776.

2. Newcomb AE, Clarke CP. Spontaneous pneumomediastinum: a benign curiosity or a significant problem? Chest 2005; 128 : 3298-3302.

3. Campillo-Soto A, Coll-Salinas A, Soria-Aledo V, Blanco-Barrio a, Flores-Pastor B, CandelARENAS M et al. Neumomediastino espontáneo: estudio descriptivo de nuestra experiencia basada en 36 casos. Arch Bronconeumol 2005; 41: 528-531.

4. Panacek EA, Singer AJ, Sherman BW, Prescott A, RUTHERFORD WF. Spontaneus pneumomediastinum: clinical and natural history. Ann Emerg Med 1992; 21: 1222-1227.

5. Toral Marín J, del Castillo Otero D, HuRtado Ayuso JE, Calderón Osuna E. Neumomediastino espontáneo como complicación de una crisis asmática. Rev Clin Esp 1999; 199: 78-80.

6. Weissberg D, Weissberg D. Spontaneous mediastinal emphysema. Eur J Cardiothorac Surg 2004; 26: 885-888.

7. Minos P, Potaris K, Gakidis I, Mazaris E, SarRas E, KonTos Z. Sports-related spontaneous pneumomediastinum. Ann Thorac Surg 2004; 78: 983-986.

8. SundaraRaghavan S, PItTS TY, SuAREZ WA, JohnSTONE C. Chest pain among adolescents with anorexia nervosa. Pediatr Emerg Care 2005; 21: 603-605.

9. Jougon JB, Ballester M, Delcambre F, Mac BRIDE T, DROMER CE, VelLy JF. Assessment of spontaneous pneumomediastinum: experience with 12 patients. Ann Thorac Surg 2003; 75: 1711-1714.

10. Koullias GJ, Korkolis DP, Wang XJ, Hammond GL. Current assessment and management of spontaneous pneumomediastinum: experience in 24 adult patients. Eur J Cardiothorac Surg 2004; 25: 852-855.

11. Freixinet J, García F, Rodríguez PM, Santana NB, Quintero CO, Hussein M. Spontaneous pneumomediastinum long-term follow-up. Respir Med 2005; 99: 1160-1163.

12. GURNEY JW, WinER-MURAM HT. Neumomediastino. En: Gurney JW, ed. Los 100 diagnósticos principales en tórax. Madrid: Elsevier España; 2004: 166-168.

13. Kaneki T, Kubo K, Kawashima A, Kolzumi T, Sekiguchi M, Sone S. Spontaneous pneumomediastinum in 33 patients: yield of chest computed tomography for the diagnosis of the mild type. Respiration 2000; 67: 408411. 